veränderbarer Legende und der Möglichkeit, Attribute von Objekten usw. abzufragen.

Gleichsam als Zugabe wurde das Vortragsprogramm spontan erweitert: Dr. Thomas Kolbe vom gastgebenden Lehrstuhl stellte, als Vergleich zu GeoMedia Web Map, kurz die Möglichkeiten von ArcIMS (Arc Internet MapServer der Fa. ESRI) dar.

Welche Basis-Fähigkeiten des Webgestaltens bereits im Grundkurs InternetKartographie vermittelt wurden, konnte ein Teilnehmer, Michael Holter von der Stadt Wuppertal, den erstaunten Zuhörern demonstrieren. Mit der Internet-Anwendung www.wuppertal.de hat er bereits Auszeichnungen gewonnen.

Besonderer Dank gilt Herrn Bernhard Horst, der es verstand, ein vielfältiges
Programm zusammenzustellen und kompetente Vortragende zu gewinnen. Auch die Organisation hatte die gewohnte, ausgezeichnete Qualität.

Insgesamt war es ein sehr lohnendes Seminar. Ziel war nicht, perfekte Entwickler von Flash-, SVG- oder Internet-GISAnwendungen zu werden, was in der Kürze der Zeit auch nicht möglich gewesen wäre. Vielmehr war die Mischung aus Theorie und Praxis dafür geeignet, genügend Einblick in die angesprochenen Themen zu geben, um sich selbst bei Bedarf später weiter darin zu vertiefen. Dazu sind das ausführliche Skriptum mit zahlreichen Links zu weiterführenden WebAdressen sowie die vielen persönlichen Kontakte zu Kolleginnen und Kollegen des Seminars bestens geeignet.

Johann Zahn, München

\title{
Neues aus der IKV
}

\section{International Cartographic Conference in Beijing, China vom 1.-10.8.2001}

Was haben Schlangen und Skorpione mit Kartographie und Geoinformation gemeinsam? Auf den ersten Blick sicherlich wenig, aber wenn die 20. International Cartographic Conference ICC in Peking statt findet, lässt sich ein zumindest kulinarischer Zusammenhang erahnen. Diese internationale Konferenz findet alle 2 Jahre statt. Nach Europa (Stockholm 1997) und Amerika (Ottawa 1999) traf man sich in diesem Jahr in Asien. Ca. 1000 Teilnehmer aus 5 Kontinenten hatten für 1 Woche schwer zu kümpfen: Fachfirmenausstellung, 3 Kartenausstellungen, Exkursionen, Vorprogramm, Nachprogramm, Kommissionssitzungen und nicht zuletzt die ca.
350 (!) Fachvorträge forderten bei $35^{\circ} \mathrm{C}$ von allen Beteiligten großes Durchhaltevermögen.

Das gastgebende Land, die Volksrepublik China, wurde bewusst gewählt: eine Öffnung zum Westen - den USA und der EU - ist zu spüren, die dem Besucher gegenübertretenden Veränderungen der letzten Jahre zeigen dies deutlich. Gleichzeitig startet China ein Programm zur Entwicklung des chinesischen Westens. Hier werden enorme Investitionen in die Infrastruktur in Gang gesetzt.

Anders als noch vor Jahren gewinnt die Stimme Deutschlands etwas mehr an Gewicht. Neben zahlreichen deutschen Vorträgen wurde die zweite von beiden Plenary Sessions maßgeblich von Frau Prof. Meng (München) und Prof. Grünreich (Frankfurt) gestaltet. Schwerpunkte der
Ausführungen waren einerseits die Globalisierung der Geoinformation und der Trend über die Landesgrenzen hinweg auf Anbieterseite zum Beispiel im Rahmen der Eurogeographics zusammen zu arbeiten. Auf der Nutzerseite hingegen geht die Entwicklung vom Massenprodukt zum Produkt on demand bzw. von der Nutzung reiner Daten zur Nutzung von persönlichen Geoservices. Auch sind nicht nur Darstellungen gefragt, die der herkömmlichen zweidimensionalen Karte ähneln, sondern "map like presentations", wobei 3D-Produkte nur eine Gruppe von vielen Möglichkeiten darstellen, "the world in your pocket" zu stecken.

Viele Beispiele in den Vorträgen zeigten diese Entwicklungen prototypisch auf. Es wurden Techniken vorgestellt, die die Zeit als vierte Dimension beinhalten, der Bereich der internetbasierenden Kartographie und Geoinformation nahm einen breiten Raum ein und eine "hörbare" Karte, eine interaktiven Anwendung, die das Erleben von Lärmbelästigungen und die Planung von positiven Geräuschkulissen zum Ziel hat, sorgte für reges Interesse.

Einige herausragende Vorträge werden übrigens, in deutscher Sprache, im Rahmen des Symposiums Kartographie 2002 vom 6.-8. Mai 2002 in Königslutter zu hören sein. (Kontakt: Bernhard Horst, Tel.0511/6479443 oder b.j.horst@ t-online.de)

Als Beispiel für gelungene Fachexkursionen kann die zum Shaanxi Provincial Bureau of Surveying and Mapping in Xi'an genannt werden. Ein Büro, hier würde man es Landesvermessungsamt nennen, das sich seiner Bedeutung und Öffentlichkeitswirksamkeit bewusst ist und seine Fähigkeiten überzeugend darstellt. Dieses Büro fungiert als besondere Instanz im Rahmen der Entwicklung des chinesischen Westens und ist - mit modernster Technik ausgestattet - auf diese Aufgaben gut vorbereitet. Hier wird die internationale Kooperation nicht nur in den Mund genommen, sondern gelebt, sowohl in wissenschaftlicher als auch in wirtschaftlicher Hinsicht.

Fazit: eine gut organisierte Veranstaltung, die mehr als nur informativ war und viele neve Ideen und Impulse für die Arbeit daheim brachte.

Ekkehard Matthias

Kommission Praktische Kartographie der DGfK Commission on Map Production of the ICA

\section{Leserforum}

Wie aus zahlreichen Beispielen in den KN hervorgeht, bemüht sich die Schriftleitung grundsätzlich, aus der Rubrik der Buchbesprechungen mehr zu machen, als nur eine bloße Vorstellung und Aneinanderreihung trockener Schilderungen von Inhalten. Eine der Meinungsfreiheit verpflichtete und lebendige Zeitschrift muss und sollte hier ein Forum für These und Antithese, für den wissenschaftlichen Diskurs mit zugespitzten Argumenten bieten und mit dieser Zielsetzung zugleich ein positives Beispiel für sachliche Auseinandersetzungen bilden, stoßen die Rezensionen doch nicht nur bei den Rezensierten, sondern in der Leserschaft generell auf erfreulich große Resonanz.

In den KN 2/2001 besprach Herr Wintges das Buch von Rita Eggert: „Urheberrechtsschutz bei Landkarten". Dazu erreichten uns folgende Zuschriften, die wir gerne an dieser Stelle veröffentlichen: 